\title{
EFFECTIVENESS ANALYSIS OF UCAV USED IN MODERN MILITARY CONFLICTS
}

\author{
Mirosław ADAMSKI (D) \\ Faculty of Aviation, Military University of Aviation, Dywizjonu 303 35, 08-521, Dęblin, Poland
}

Received 15 December 2019; accepted 31 January 2020

\begin{abstract}
Analysis of contemporary armed conflicts shows that UCAV (Unmanned Combat Air Vehicle) is finding an increasing range of combat applications. The present study deals with constructional characteristics, tactical and technical parameters, equipment with reconnaissance sensors, electronic warfare equipment, weaponry, economic coefficient, combat experience, possessed additional benefits, systems and technologies, performance and application options in combat operations. Two UCAV (MALE - Medium Altitude Long Endurance) classes were analyzed based on Heron, Heron TP, MQ-1B Predator, MQ-1C Gray Eagle, Wing Loong, CH-4B and newly introduced MQ-9 Reaper ER, MQ-9 Reaper, P.1HH HammerHead, Mantis and (HALE - High Altitude Long Endurance) Global Yabhon, Yabhon-United 40 to determine their optimal effectiveness in combat operations. The article presents a general methodology for assessing the tactical effectiveness of selected UAV classes that are or can be used in modern armed conflicts. It can be useful for potential interested parties when making decisions regarding the purchase or application of an appropriate UAV depending on the capabilities and conditions of the defense strategy of a given country.
\end{abstract}

Keywords: aviation, missile, UAV, combat, analysis.

\section{Introduction}

The analysis of the armed conflicts of the last 20 years indicates that the importance of UAVs in the modern world has grown significantly. The nature of these conflicts, often asymmetric and against irregular forces, shows how useful in the modern battlefield UAVs have become.

The analysis was carried out using the statistical and comparative methods. In order to determine and compare the application possibilities of selected UCAVs, an analysis was performed in terms of their performance, reconnaissance and electronic warfare capabilities, impact/strike capabilities, combat experience, economic coefficient and additional combat capabilities.

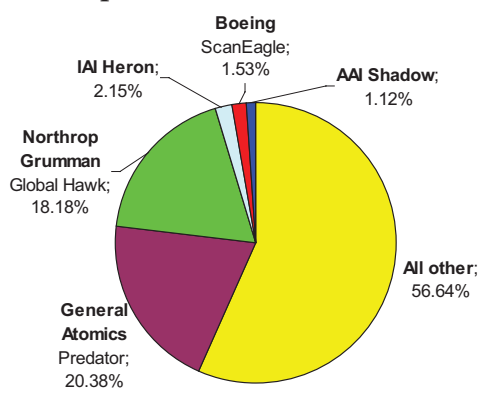

Figure 1. The largest manufacturers of UAVs and UCAVs in the world (source: own study)
The demand for unmanned aerial vehicles in the last decade grew bigger and bigger, but the last few years one can see signs of a slowdown due to steadily shrinking arms market and funding cuts for the reinforcement made by the governments of NATO members (Figure 1 and 2).

The UCAV class together with the HALE and MALE is currently $55 \%$ of the global market, which gives it the first position on the global UAV market. However, the important fact is that a large part of TUAV (Tactical UAV) also have the ability to carry armament. The main purpose of the article is to determine the highest efficiency

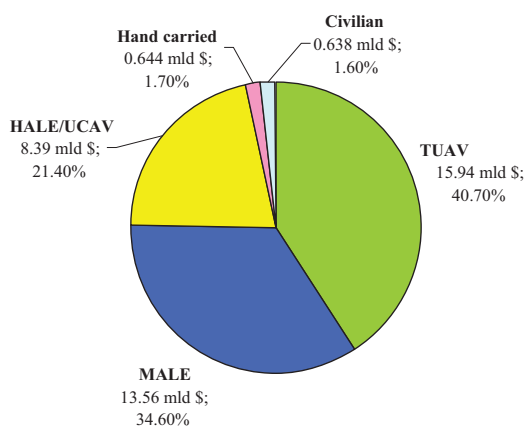

Figure 2. The division of the global UAV market in terms of the demand for a particular class (source: own study)

*Corresponding author. E-mail: tetrazen@wp.pl 
of UCAV during the ISTAR (Intelligence, Surveillance, Target Acquisition, Reconnaissance) and SEAD (Suppresion of Enemy Air Defenses) missions.

\section{Method of analysis}

For the purpose of the analysis, statistical UCAV was determined for both classes. The statistical UCAV has been described using the average performance parameters (arithmetic average) and has been assigned equipment that should be of a medium-class combat UAV, including combat experience and a convenient airframe price.

With reference to the statistical UCAV, the formulas were derived for the performance coefficient $-W_{O}$, the reconnaissance and electronic warfare capabilities coefficient $-W_{R}$, the impact/strike capabilities coefficient $-W_{U}$ and the coefficient covering combat experience, economic coefficient plus additional advantages and systems of a given UCAV - $W_{D E Z}$. Based on these, the formula was derived for the aggregate coefficient of combat capabilities $W_{U C}$. It was used for final classification of UCAVs analyzed in terms of possible applications in combat operations.

The first analyzed parameter is the performance coefficient $W_{O}$. It is a unitless, point factor that includes parameters such as service ceiling, flight endurance, maximum airspeed and relation between MUW (Maximum Useful Weight) and MTOW (Maximum Take Off Weight). It was based on the principle of proportion in order to obtain scaled point values relative to the statistical UCAV $\left(A_{S}\right.$, $E_{S}, v_{S}, Z m_{S}$ was defined as 100 points) and an arithmetic average of:

$$
W_{O}=\frac{\frac{A \cdot 100}{A_{S}}+\frac{E \cdot 100}{E_{S}}+\frac{v \cdot 100}{v_{S}}+\frac{Z m \cdot 100}{Z m_{S}}}{4},
$$

after simplification, the formula for the $W_{O}$ coefficient was obtained as:

$$
W_{O}=25 \cdot\left(\frac{A}{A_{S}}+\frac{E}{E_{S}}+\frac{v}{v_{S}}+\frac{Z m}{Z m_{S}}\right),
$$

where: $W_{O}$ - UCAV's performance coefficient; $A$ - UCAV's ceiling; $E$ - UCAV's flight endurance; $v$ - UCAV's maximum airspeed; $Z m$ - UCAV's MUW to MTOW relation; $A_{S}$ - statistical UCAV's ceiling; $E_{S}$ - statistical UCAV's flight endurance; $v_{S}$ - statistical UCAV's maximum airspeed; $Z m_{S}$ - statistical UCAV's MUW to MTOW relation.

Second formula was derived for the UCAV reconnaissance and electronic warfare capabilities coefficient $W_{R}$. It is a unitless, point factor, covering variety of sensors and MUW parameters. It was based on the principle of proportion in order to obtain scaled point values relative to the statistical UCAV ( $\sum_{R S}$ was defined as 100 points) and the weighted average formula in which the weights were granted as follows:

1) variety of sensors - weight equal to 90 ,

2) MUW - weight equal to 10.

The weights were assigned in such a proportion, because in terms of reconnaissance possibilities, the quality and multi-variant of the sensors have greater impact on the effectiveness than the payload.

$$
W_{R}=\frac{90 \cdot \frac{\sum_{R} \cdot 100}{\sum_{R S}}+10 \cdot \frac{m_{M U W} \cdot 100}{m_{M U W S}}}{90+10},
$$

after simplification, the formula for the $W_{R}$ coefficient was obtained as:

$$
W_{R}=10 \cdot\left(\frac{9 \sum_{R}}{\sum_{R S}}+\frac{m_{M U W}}{m_{M U W S}}\right),
$$

where: $W_{R}$ - UCAV's reconnaissance and electronic warfare capabilities coefficient; $\Sigma_{R}$ - sum of points granted to UCAV for multi-variant sensors; $m_{M U W}$ - UCAV's maximum useful weight; $\Sigma_{R S}$ - sum of points granted to statistical UCAV for multi-variant sensors; $m_{M U W S}$ - statistical UCAV's maximum useful weight.

Another coefficient is based on the UCAV impact/ strike capabilities and is marked as $W_{U}$. It is a unitless, point factor that includes parameters such as multivariant armament, number of hardpoints and maximum payload. It has been based on the principle of proportion in order to obtain scaled point values related to the statistical UCAV ( $\Sigma_{U S}$ was defined as 100 points) and the weighted average formula in which the weights were granted as follows:

3) multi-variant armament - weight equal to 20 ,

4) number of hardpoints - weight equal to 10 ,

5) MUW - weight equal to 70.

The weights were assigned in such a proportion, because in terms of impact/strike capabilities, attention should be paid to the quantity and quality of the carried armament. Hence, the $W_{U}$ coefficient puts special emphasis on MUW, since a large payload allows the simultaneous carriage of large amounts of munitions (depending on the number of hardpoints). The multi-variant of the armaments were put on a further plan because MALE and HALE are reconnaissance-striking aircraft designed primarily to destroy small or medium ground and surface targets and are currently unable to withstand the direct air combat with much larger, high performance multirole combat aircraft.

$$
W_{U}=\frac{20 \cdot \frac{\sum_{U} \cdot 100}{\sum_{U S}}+10 \cdot \frac{P_{P} \cdot 100}{P_{P S}}+70 \cdot \frac{m_{M U W} \cdot 100}{m_{M U W S}}}{20+10+70},
$$

after simplification, the formula for the $W_{U}$ coefficient was obtained as:

$$
W_{U}=10 \cdot\left(\frac{7 m_{M U W}}{m_{M U W S}}+\frac{2 \sum_{U}}{\sum_{U S}}+\frac{P_{P}}{P_{P S}}\right),
$$

where: $W_{U}$-UCAV's impact/strike capabilities coefficient; $\Sigma_{U}$ - sum of points granted to UCAV for multi-variant armament; $m_{M U W}$ - UCAV's maximum useful weight; $P_{P}$ - number of UCAV's hardpoints; $\Sigma_{U S}$ - sum of points granted to statistical UCAV for multi-variant armament; $m_{M U W S}$ - statistical UCAV's maximum useful weight; $P_{P S}$ - number of statistical UCAV's hardpoints. 
The next parameter taken into account in the analysis is the $W_{D E Z}$ coefficient, which includes the combat experience of a given UCAV, its economic coefficient and individual additional advantages and equipment. $W_{D E Z}$ is an estimation coefficient, which for the purposes of statistical analysis has been reduced to a numerical value, by granting points to each UCAV according to the following rule:

1) combat experience $0-50$ points;

2) economic coefficient:

a) estimated cost of one UCAV airframe:

- less than $\$ 1$ million - 55 points,

- over $\$ 50$ million -5 points.

3) additional advantages and systems:

a) each additional equipment -5 points,

b) each additional doubled system -6 points.

It should be noted, however, that the points granted in individual parameters of $W_{D E Z}$ coefficients are approximate and estimated values.

Finally, the formula was derived for the coefficient of UCAV applicability in combat operations $-W_{U C}$. It is a unitless, point factor that includes within its scope four above-mentioned factors, defining the possibilities of applications in combat operations. It was based on the arithmetic average of $W_{O}, W_{R}, W_{U}, W_{D E Z}$ coefficients.

$$
W_{U C}=\frac{W_{O}+W_{R}+W_{U}+W_{D E Z}}{4} .
$$

With the above statistical comparison method, it was possible to identify which of the UCAVs analyzed has optimum application efficiency in the combat operations.

\section{Analysis of the effectiveness of combat application}

To analyze the possibilities of application of UCAVs in combat operations, 6 MALE class, 4 MALE new generation and 2 HALE class have been selected. The main tasks facing this class of UCAV are missions performed for all types of armed forces in the field of ISTAR, long range with long surveillance duration, precise fire support, SEAD identification and immediate control of ground, surface, underwater and air targets, data retransmission and data transfer from the battlefield in near real time.

\subsection{Comparison of the performance of selected UCAVs}

The analysis of application possibilities in combat operations launched from comparison of parameters such as ceiling, flight endurance, maximum airspeed and the MUW to MTOW realation (Table 1 and 2).

\subsection{Comparison of reconnaissance and electronic warfare capabilities}

One of the main capabilities of UCAV is the carriage of reconnaissance equipment. It is the mission of ISR (Intelligence, Surveillance, Reconnaissance) the first UAVs were designed years ago. Currently, their scope of activities has been extended with the possibility of electronic warfare, which together with reconnaissance capabilities, is another point of analysis (Table 3 ).

\subsection{Comparison of impact/strike capabilities}

The foremost ability from the subject of analysis point of view is the mass, number and type of armament carried by UCAV, thanks to which they gain impact/strike capabilities (Table 4).

\subsection{Comparison of combat experience, economic coefficient and additional advantages and systems of selected UCAVs}

The last coefficient necessary to calculate $W_{U C}$ is the $W_{D E Z}$ factor. It includes: combat experience, economic coefficient and additional advantages and systems of UCAV (Table 5).

Table 1. Performance of selected MALE and HALE class UCAVs (source: own study)

\begin{tabular}{|c|c|c|c|c|}
\hline & Ceiling [ft] & Flight Endurance [h] & Maximum Airspeed [kt] & $\begin{array}{l}\text { MUW to MTOW } \\
\text { relation }[\mathrm{kg}]\end{array}$ \\
\hline $\mathrm{CH}-4 \mathrm{~B}$ & 23000 & 14 & 211 & 0.259 \\
\hline Global Yabhon & 50000 & 40 & 740 & 0.330 \\
\hline Heron & 30000 & 45 & 222 & 0.315 \\
\hline Heron TP & 45000 & 36 & 209 & 0.215 \\
\hline Mantis & 50000 & 36 & 555 & 0.178 \\
\hline MQ-1B Predator & 25000 & 40 & 222 & 0.294 \\
\hline MQ-1C Gray Eagle & 29000 & 25 & 309 & 0.299 \\
\hline Improved Gray Eagle & 29000 & 50 & 309 & 0.384 \\
\hline MQ-9 Reaper & 50000 & 37 & 444 & 0.367 \\
\hline MQ-9 Reaper ER & 50000 & 42 & 444 & 0.509 \\
\hline P.1HH HammerHead & 45000 & 16 & 731 & 0.309 \\
\hline Wing Loong & 23000 & 20 & 281 & 0.167 \\
\hline Yabhon-United 40 & 26000 & 100 & 200 & 0.525 \\
\hline Statistical MALE and HALE class UCAV & 36538 & 38.54 & 374 & 0.319 \\
\hline
\end{tabular}


Table 2. MALE and HALE class UCAV classification by $W_{O}$ (source: own study)

\begin{tabular}{|c|l|c|}
\hline Position & \multicolumn{1}{|c|}{ MALE and HALE class UCAV } & $W_{O}[\mathrm{pts}]$ \\
\hline 1. & Yabhon-United 40 & 137.08 \\
\hline 2. & Global Yabhon & 135.31 \\
\hline 3. & MQ-9 Reaper ER & 130.90 \\
\hline 4. & MQ-9 Reaper & 116.54 \\
\hline 5. & P.1HH HammerHead & 114.06 \\
\hline 6. & Mantis & 108.49 \\
\hline 7. & Improved Gray Eagle & 102.93 \\
\hline N/A & Statistical MALE-HALE class UCAV & 100.00 \\
\hline 8. & Heron & 89.18 \\
\hline 9. & Heron TP & 84.91 \\
\hline 10. & MQ-1B Predator & 80.87 \\
\hline 11. & MQ-1C Gray Eagle & 80.06 \\
\hline 12. & Wing Loong & 60.53 \\
\hline 13. & CH-4B & 59.15 \\
\hline
\end{tabular}

Table 3. MALE and HALE class UCAV classification by $W_{R}$ (source: own study)

\begin{tabular}{|c|l|c|}
\hline Position & \multicolumn{1}{|c|}{ MALE and HALE class UCAV } & $W_{R}[\mathrm{pts}]$ \\
\hline 1. & MQ-9 Reaper ER & 176.50 \\
\hline 2. & MQ-9 Reaper & 168.66 \\
\hline 3. & Heron TP & 123.94 \\
\hline 4. & Improved Gray Eagle & 121.74 \\
\hline 5. & MQ-1C Gray Ealge & 119.73 \\
\hline 6. & Heron & 119.01 \\
\hline 7. & P.1HH HammerHead & 118.49 \\
\hline 8. & MQ-1B Predator & 105.65 \\
\hline N/A & Statistical MALE-HALE class UCAV & 100.00 \\
\hline 9. & Wing Loong & 78.79 \\
\hline 10. & Mantis & 77.45 \\
\hline 11. & Yabhon-United 40 & 72.92 \\
\hline 12. & CH-4B & 67.12 \\
\hline 13. & Global Yabhon & 65.72 \\
\hline
\end{tabular}

\section{Analysis results}

Based on the analysis (Table 6), it was concluded that UCAV with the best application possibilities in combat operations is MQ-9 Reaper ER and MQ-9 Reaper (Figure 3).

Both American constructions came off very well in terms of all coefficients (MQ-9 Reaper ER: $W_{O}$ - position. $3, W_{R}-$ pos. $1, W_{U}$ - pos. 2 , and $W_{D E Z}-$ pos. 5 ; MQ-9 Reaper: $W_{O}$ - pos. $4, W_{R}-$ pos. $2, W_{U}-$ pos. 3 , and $W_{D E Z}$ - pos. 1). The biggest difference, between the two UCAVs produced by GA-ASI can be observed in the case of impact/strike capabilities, and although both versions have very wide multi-variant weaponry potential, thanks to 6 suspension nodes and greater MUW, the MQ-9 Reaper ER owes its better result to the older version.
Table 4. MALE and HALE class UCAV classification by $W_{U}$ (source: own study)

\begin{tabular}{|c|l|c|}
\hline Position & \multicolumn{1}{|c|}{ MALE and HALE class UCAV } & $W_{U}[\mathrm{pts}]$ \\
\hline 1. & Global Yabhon & 280.68 \\
\hline 2. & MQ-9 Reaper ER & 261.86 \\
\hline 3. & MQ-9 Reaper & 202.00 \\
\hline 4. & P.1HH HammerHead & 131.52 \\
\hline 5. & Mantis & 127.11 \\
\hline 6. & Yabhon-United 40 & 118.66 \\
\hline N/A & Statistical MALE-HALE class UCAV & 100.00 \\
\hline 7. & Heron TP & 71.14 \\
\hline 8. & Improved Gray Eagle & 57.85 \\
\hline 9. & Wing Loong & 53.66 \\
\hline 10. & MQ-1C Gray Ealge & 43.81 \\
\hline 11. & CH-4B & 41.29 \\
\hline 12. & Heron & 36.60 \\
\hline 13. & MQ-1B Predator & 33.14 \\
\hline
\end{tabular}

Table 5. MALE and HALE class UCAV classification by $W_{D E Z}$ (source: own study)

\begin{tabular}{|c|l|c|}
\hline Position & \multicolumn{1}{|c|}{ MALE and HALE class UCAV } & $W_{D E Z}[\mathrm{pkt}]$ \\
\hline 1. & MQ-9 Reaper & 140 \\
\hline 2. & MQ-1C Gray Ealge & 135 \\
\hline 3. & Heron & 127 \\
\hline 4. & MQ-1B Predator & 126 \\
\hline 5. & MQ-9 Reaper ER & 120 \\
\hline 6. & Heron TP & 102 \\
\hline N/A & Statistical MALE-HALE class UCAV & 100 \\
\hline 7. & Improved Gray Eagle & 100 \\
\hline 8. & P.1HH HammerHead & 87 \\
\hline 9. & Wing Loong & 85 \\
\hline 10. & CH-4B & 75 \\
\hline 11. & Yabhon-United 40 & 50 \\
\hline 12. & Mantis & 40 \\
\hline 13. & Global Yabhon & 20 \\
\hline
\end{tabular}

Third position in the ranking took the biggest, yet still being in the phase of design Global Yabhon, which is characterized by enormous impact/strike capabilities. If the manufacturer's announcements from the United Arab Emirates confirm the assumed performance, then the construction of ADCOM Systems can be called an unmanned bomber.

P.1HH HammerHead was classified in the fourth position, obtaining the result almost $13 \%$ better than statistical MALE and HALE class UCAV. It is an Italian construction designed by Piaggio Aero, still in the testing phase. P.1HH HammerHead is an equally balanced unit like the MQ-9 Reaper is, with the difference, however, that in the values of all analyzed coefficients it came off worse than the American competitor, taking respectively the $5^{\text {th }}$ position in the $W_{O}$ classification, $7^{\text {th }}$ in $W_{R}, 4^{\text {th }}$ in $W_{U}$ and $8^{\text {th }}$ in $W_{D E Z}$. 
Table 6. MALE and HALE class UCAV classification by $W_{U C}$ (source: own study)

\begin{tabular}{|c|l|c|}
\hline Position & \multicolumn{1}{|c|}{ MALE and HALE class UCAV } & $W_{U C}[\mathrm{pts}]$ \\
\hline 1. & MQ-9 Reaper ER & 172.31 \\
\hline 2. & MQ-9 Reaper & 156.80 \\
\hline 3. & Global Yabhon & 125.43 \\
\hline 4. & P.1HH HammerHead & 112.77 \\
\hline N/A & Stat. UCAV kl. MALE-HALE & 100.00 \\
\hline 5. & Improved Gray Eagle & 95.63 \\
\hline 6. & Heron TP & 95.50 \\
\hline 7. & Yabhon-United 40 & 94.66 \\
\hline 8. & MQ-1C Gray Ealge & 94.65 \\
\hline 9. & Heron & 92.95 \\
\hline 10. & Mantis & 88.26 \\
\hline 11. & MQ-1B Predator & 86.42 \\
\hline 12. & Wing Loong & 69.49 \\
\hline 13. & CH-4B & 60.64 \\
\hline
\end{tabular}

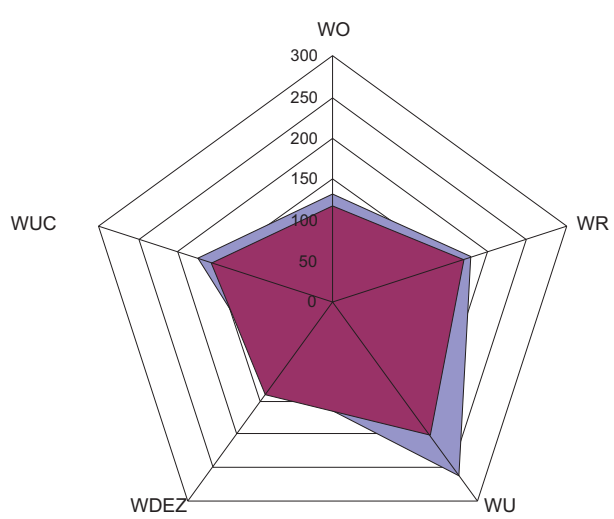

$\square$ MQ-9 Reaper ER $\square$ MQ-9 Reaper

Figure 3. Comparison of MQ-9 Reaper ER and MQ-9 Reaper by $W_{O}, W_{R}, W_{U}, W_{D E Z}, W_{U C}$ coefficients (source: own study)

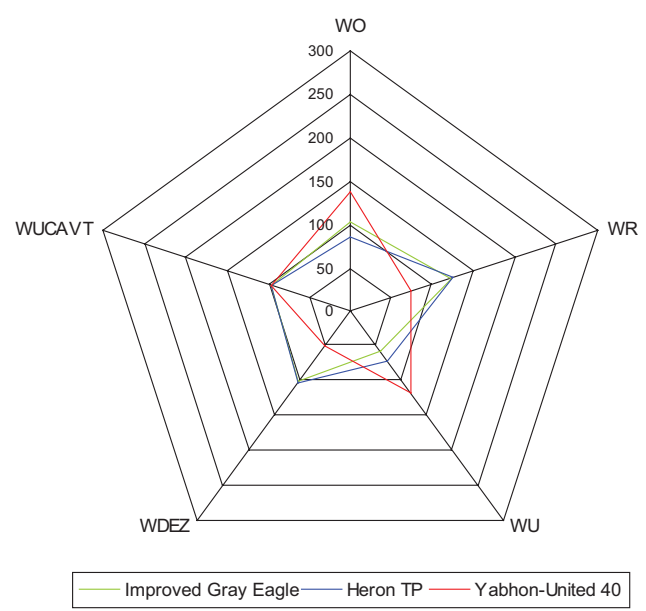

Figure 4. Comparison of Improved Gray Eagle, Heron TP and Yabhon-United by $W_{O}, W_{R}, W_{U}, W_{D E Z}, W_{U C}$ coefficients (source: own study)

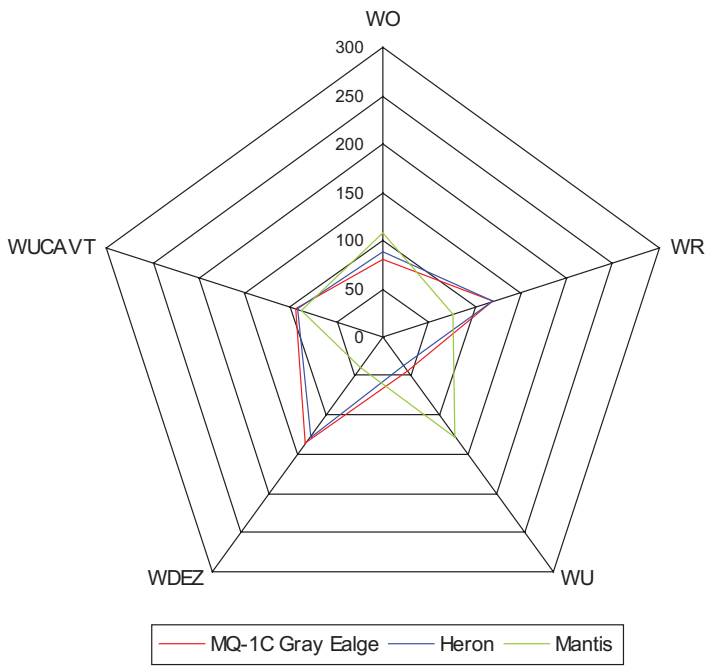

Figure 5. Comparison of MQ-1C Gray Eagle, Heron and Mantis by $W_{O}, W_{R}, W_{U}, W_{D E Z}, W_{U C}$ coefficients (source: own study)

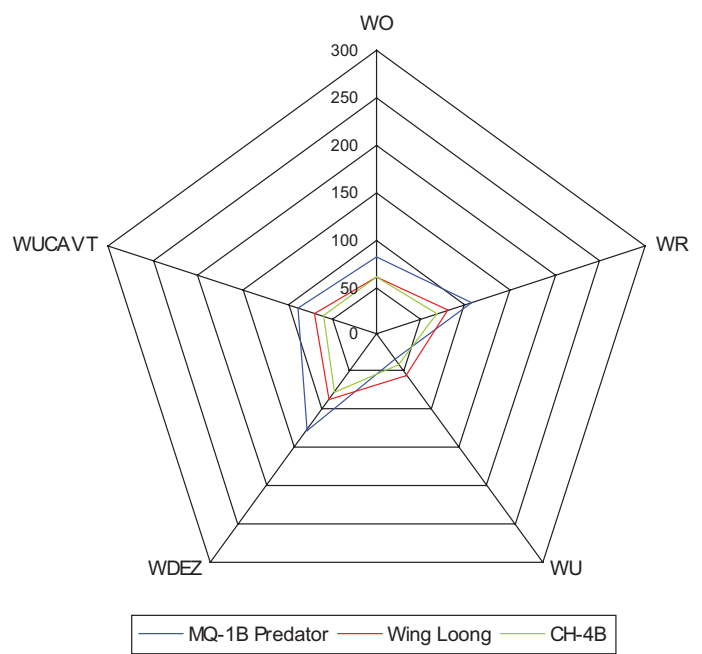

Figure 6. Comparison of MQ-1B Predator, Wing Loong i CH$4 \mathrm{~B}$ by $W_{O}, W_{R}, W_{U}, W_{D E Z}, W_{U C}$ coefficients (source: own study)

The 5 th to 9 th positions were taken by IGE, Heron TP (the two constructions are balanced, they were solid in each factor), Yabhon-United 40 (construction focused on long flight endurance and good strike capabilities, including against the submarines), MQ-1C Gray Eagle and Heron (both structures geared to high quality reconnaissance, in the case of MQ-1C also electronic warfare, having a significant battle experience and a large number of additional benefits and systems). The minimal differences between these five UAV's indicate that they have very similar combat operations (Figure 4 and 5).

The 10th position, with the result of almost $12 \%$ worse than the statistical MALE and HALE class UCAV, was taken by Mantis. The BAE Systems construction has a very large impact/strike potential, as well as reconnaissance, if only they implement onboard technologies that British already have. The MQ-1B Predator obtained 14\% lower score 
than the statistical MALE and HALE class UCAV. This is the most common UCAV design with the greatest combat experience, which, however, is getting a bit outdated. In its place GA-ASI has already created similar size MQ$1 \mathrm{C}$ Gray Eagle ( $8^{\text {th }}$ position) and larger MQ-9 Reaper $\left(2^{\text {nd }}\right.$ position), as well as their modernization (IGE $-5^{\text {th }}$ position and MQ-9 Reaper ER - $1^{\text {st }}$ position), which are slowly replacing the worn-out MQ-1B Predator. Wing Loong and $\mathrm{CH}-4 \mathrm{~B}$ were classified in the last two positions. Both constructions were supposed to be a Chinese response to the American MQ-1B Predator and MQ-9 Reaper. Analyzing the airframes of American and Chinese UCAV concluded that designing their drones, Chinese were strongly inspired by the MQ-1B Predator design scheme. However, at the moment neither Wing Loong nor $\mathrm{CH}-4 \mathrm{~B}$ can not match the range of their capabilities in combat operations with the latest American constructions (Figure 6).

\section{Conclusions}

After the analysis, the hypothesis regarding the current and future domination of the USA and Israel in the entire market of unmanned combat aerial vehicles was partly confirmed. In terms of performance and potential applications in combat operations, the Israeli place on the defense market is being tackled by producers from the Arab Emirates and Europe.

Structures originating from Israel are still used by many armies of the world, however, they are beginning to increasingly stand out with their parameters and capabilities from currently being developed. The constructors from ADCOM Systems want to mark their presence on the defense market particularly strongly. Their UCAVs surprise with their innovativeness, impact/strike capabilities and performance, although due to the lack of appropriate technological advancement, Arabic constructions have fairly average reconnaissance capabilities. Europe is also trying to catch up with losses incurred during the early years of the 21 st century, trying to bring to the market very promising designs such as P.1HH HammerHead and Mantis.

Summing up, it should be stated that combat unmanned aerial vehicles are widely used in combat operations, they share an increasingly significant part of the armaments sector and have a real impact on the course of armed conflicts. Their increasing performance and combat capabilities allow for efficient replacement of older generations of manned combat aircraft. In addition, UCAV have a strong, almost irreplaceable position performing tasks with an increased risk of shooting down, such as SEAD missions and missions during conflicts with irregular forces (for example, the fight against terrorism) not requiring the involvement of conventional air force, the use of which is associated with high costs.

\section{Disclosure statement}

I do not have any competing financial, professional, or personal interests from other parties.

\section{References}

Adamski, M., \& Rajchel, J. (2013). Bezzałogowe statki powietrzne (cz. 1). Wyższa Szkoła Oficerska Sił Powietrznych, Dęblin.

Adamski, M., Vogt, R., \& Ćwiklak, J. (2014). Integrated navigation and pilotage systems. Paper presented at the IEEE Chinese Guidance, Navigation and Control Conference. https://doi.org/10.1109/CGNCC.2014.7007341

Biass, E. H., \& Braybrook, R. (2012). Compedium drones 2012. Armada, 3.

Cwojdziński, L., \& Adamski, M. (2014). Power units and power supply systems in UAV. Aviation, 18(1), 1-8. https://doi.org/10.3846/16487788.2014.865938

Derbel, K., \& Beneda, K. (2019). Linear dynamic mathematical model and identification of micro turbojet engine for Turbofan Power Ratio control. Aviation, 23(2), 54-64. https://doi.org/10.3846/aviation.2019.11653

Hansen, B. (2009). Unmanned Aircraft Systems present \& future capabilities. Washington.

Headquarters, United States Air Force. (2009). United States Air Force Unmanned Aircraft Systems Flight Plan 2009-2047. Washington.

Kulyk, M., Kharchenko, V., \& Matiychyk, M. (2011). Justification of thrust vector deflection of twin-engine unmanned aerial vehicle power plants. Aviation, 15(1), 25-29. https://doi.org/10.3846/16487788.2011.566319

Oktay, T., Uzun, M., \& Kanat, O. O. (2018). Maximum lift/drag ratio improvement of TUAVs via small aerodynamic modifications. Aircraft Engineering and Aerospace Technology, 90, 1438-1444. https://doi.org/10.1108/AEAT-07-2017-0175

Skinder, T. (2005). ADCOM unveils new HALE UAV. Unmmaned Vehicles, 18.

Sochacki, A. (2014). Analiza porównawcza możliwości zastosowań $w$ działaniach bojowych bezzałogowych statków powietrznych klasy UCAV (praca mgr). Wyższa Szkoła Oficerska Sił Powietrznych.

Valavanis, K. P. (2008). Advances in unmanned aerial vehicles: state of the art the road to autonomy. University of South Florida Tampa, USA. https://doi.org/10.1007/978-1-4020-6114-1

Warwick, G., \& Dickerson, L. (2012). Cooling down? Aviation Week \& Space Technology, 174, 80-84.

Wise, K. A. (2003). First flight of the X-45A Unmanned Combat Air Vehicle (UCAV). Paper presented at the AIAA Atmospheric Flight Mechanics Conference and Exhibit. https://doi.org/10.2514/6.2003-5320

Wyatt, E. C., \& Hirschberg, M. J. (2003). Transforming the future battlefield: the DARPA/Air force Unmanned Combat Air Vehicle (UCAV) program. Paper presented at the AIAA International Air and Space Symposium and Exposition: the Next 100 Years. https://doi.org/10.2514/6.2003-2616 